\title{
Meta
}

Journal des traducteurs

Translators' Journal

\section{TYMOCZKO, MARIA (2007) : Enlarging Translation, Empowering Translators. Manchester: St. Jerome Pulblishing, 353 p.}

\section{Eve-Marie Gendron-Pontbriand}

Volume 58, numéro 1, avril 2013

URI : https://id.erudit.org/iderudit/1023819ar

DOI : https://doi.org/10.7202/1023819ar

Aller au sommaire du numéro

Éditeur(s)

Les Presses de l’Université de Montréal

ISSN

0026-0452 (imprimé)

1492-1421 (numérique)

Découvrir la revue

Citer ce compte rendu

Gendron-Pontbriand, E.-M. (2013). Compte rendu de [TYMOczKo, MARIA

(2007) : Enlarging Translation, Empowering Translators. Manchester :

St. Jerome Publishing, 353 p.] Meta, 58(1), 247-250.

https://doi.org/10.7202/1023819ar d'utilisation que vous pouvez consulter en ligne.

https://apropos.erudit.org/fr/usagers/politique-dutilisation/ 


\section{DOCUMENTATION}

\section{Comptes rendus}

Tymoczko, Maria (2007): Enlarging Translation, Empowering Translators. Manchester: St. Jerome Publishing, 353 p.

Dans son plus récent livre, Enlarging Translation, Empowering Translators, Tymoczko prône une reconceptualisation de la traduction visant à intégrer une plus grande diversité de points vue quant à sa théorisation et à sa pratique; ceci mènerait, selon elle, à l'affranchissement des traducteurs et favoriserait leur pouvoir d'agir ${ }^{1}$.

Dans la première partie du livre, l'auteure aborde la traduction en tant que concept théorique, qu'elle cherche à redéfinir et à élargir. Le premier chapitre se veut un survol historique des fondements théoriques et épistémologiques de la traductologie. Elle oriente d'ailleurs sa présentation par rapport au positivisme. Tymoczko fait valoir que la Deuxième Guerre mondiale a engendré un changement de paradigme épistémologique. Elle explique que, pour des raisons stratégiques, les puissances belligérantes se sont particulièrement intéressées aux différentes langues et cultures, notamment dans le but de décoder les messages ennemis et d'utiliser efficacement la propagande. Ces recherches ont mis à jour l'absence de réalités objectives facilement observables, particulièrement en ce qui a trait aux lettres et aux sciences sociales, minant ainsi la crédibilité du courant positiviste. Après la fin de la guerre, les études en traductologie se sont donc poursuivies selon une orientation décidément postpositiviste, ayant toutes en commun la reconnaissance et la valorisation de la multiplicité des perspectives. L'auteure souligne notamment les contributions des approches linguistiques et fonctionnelles, ainsi que celles des Etudes descriptives (Descriptive Translation Studies), notamment le tournant culturel (cultural turn) le tournant du pouvoir (power turn) et le tournant international (international turn). Elle passe également en revue la traduction postcoloniale, la traduction féministe, la traduction poststructuraliste et la déconstruction.

Selon Tymoczko, une perspective postpositiviste doit s'accompagner d'une redéfinition du concept même de traduction. En effet, elle fait valoir que la traduction a traditionnellement été envisagée comme le transfert fidèle d'un sens préexistant au texte, mais que cette conception se base largement sur l'expérience de la traduction biblique; ainsi, elle est le fruit d'une histoire et d'une idéologie proprement occidentale. Pourtant, la traduction a été conceptualisée et pratiquée de multiples façons différentes de par le monde et à travers les âges, comme le démontre Tymoczko avec de nombreux exemples issus d'Afrique, d'Inde, de Chine, du monde arabe et des îles d'Asie du Sud-Est. Pour cette raison, l'auteure affirme que la traduction est un concept interculturel, intertemporel et interlinguistique. En effet, on ne peut identifier de traits sémantiques minima permettant de décrire toutes les traductions tout en excluant toutes les non-traductions. Tymoczko adopte donc la définition énoncée par Gideon Toury: on nomme «traduction» tout texte considéré comme tel dans la culture cible. Elle continue sur ce point en déclarant que la traduction constitue un concept en grappe (cluster concept), terme adapté des travaux de Ludwig Wittgenstein. Les frontières des concepts en grappe sont floues et changeantes. Ainsi, tous les types de traduction recensés dans le monde partagent de nombreuses similitudes partielles et chevauchantes, sans qu'on puisse déterminer un ou plusieurs traits définitoires stricts; ils sont néanmoins unis par une certaine "ressemblance de famille». De plus, l'appartenance à un concept en grappe n'est pas déduite logiquement, mais plutôt selon l'usage; elle dépend d'une observation et d'une caractérisation a postériori, et non d'une stipulation ou d'une définition a priori. Selon Tymoczko, une telle redéfinition de la traduction - profondément postpositiviste - permettrait à la traductologie de dépasser son cadre eurocentrique pour devenir une discipline réellement représentative de la diversité internationale en fait de traduction.

Bien que la définition de Toury soit inclusive, Tymoczko juge qu'elle en dit peu sur la substance du concept en grappe *traduction ${ }^{2}$. Au troisième chapitre, l'auteure l'explore donc plus en profondeur en adoptant la stratégie suivante: utiliser comme cadres de référence des concepts qui lui sont superordonnés. Tymoczko résume la pertinence de l'utilisation de cadre de référence comme suit:

[They] illuminate the nature of specific clusters or types of translation... [They] show the relationships that different clusters of translation have with each other within the larger category *translation and the ways specific translations are linked through partial and overlapping correspondences related to these modes. (Tymoczko 2007: 111) 
Pour sa propre recherche, l'auteure a choisi des concepts généraux qu'elle qualifie de modes d'interface culturelle: la représentation, la transmission et la transculturation. Premièrement, la majorité des traductions peuvent être envisagées comme des représentations. En effet, elles constituent une image de leur original. En tant que représentation, elles peuvent ainsi construire un portrait concret d'une culture source dans l'imaginaire d'un public cible, pour le meilleur ou pour le pire. Deuxièmement, la plupart des traductions impliquent le transfert d'éléments du texte source vers le texte cible (sens, éléments formels, fonction, etc.). Or, les critères de transfert varient largement d'une culture à l'autre: certaines favorisent le transfert complet et fidèle, d'autres admettent un transfert presque nul. Bien que le paradigme eurocentrique dominant ait toujours préféré la première option, l'auteure estime que tous les degrés de transfert doivent être pris en compte par la traductologie. Troisièmement, la traduction peut aussi être une forme de transculturation (terme que Tymozcko attribue à Fernando Ortiz, 1940), un processus caractérisé par l'échange d'éléments culturels entre deux cultures. Contrairement à la transmission, on ne parle de transculturation que lorsque les éléments culturels étrangers sont naturalisés et incorporés au sein de la culture réceptrice. Selon Tymoczko, l'usage de ces trois cadres de référence dans l'étude du concept en grappe *traduction favoriserait l'autoréflexion des chercheurs et leur permettrait de dépasser leur conception personnelle de la traduction.

Un tel remaniement du discours en traductologie doit, selon l'auteur, se répercuter sur les méthodologies de recherche, qu'elle aborde au chapitre suivant. De son avis, la majorité des traductologues n'ont pas nécessairement été formés en tant que chercheur. Or, une bonne maîtrise de la méthode scientifique contemporaine, reformulée à la lumière du postpositivisme, est essentielle pour l'atteinte de conclusions valides et vérifiables. L'auteure prend donc soin de décrire la méthode scientifique, de la collecte de données à la formulation de conclusions, pour ensuite examiner tour à tour cinq de ses éléments cruciaux dans le contexte spécifique de la recherche en traductologie. Elle aborde d'abord le problème d'une classification efficace, qui selon elle forme la base de toute théorie. L'auteure rappelle que, puisqu'elle traite d'un concept en grappe interculturel, intertemporel et interlinguistique, la traductologie nécessite un système de classification souple et représentatif des multiples manifestations de la traduction dans le monde. Deuxièmement, Tymoczko discute des outils de validation qu'elle estime les plus appropriés pour la discipline, incitant d'ailleurs les traductologues à recourir le plus souvent possible à des méthodes quantitatives. Elle soutient également que la convergence des conclusions obtenues à différents paliers textuels peut faire office de validation, par exemple lorsqu'une analyse contextuelle confirme les résultats obtenus grâce à des micro-analyses linguistiques. Troisièmement, l'auteure somme les traductologues de reconnaître l'interdépendance entre théorie et plan de recherche. En effet, la collecte, la description et l'analyse de données, ainsi que la formulation d'hypothèses et de conclusions, tous ces processus ne sont pas neutres mais plutôt basés sur une approche théorique. C'est pourquoi elle encourage l'introspection chez les chercheurs, pour qu'ils puissent identifier leurs présupposés théoriques et leurs partis pris. Quatrièmement, elle discute de la nature des expériences pouvant être réalisées en traductologie. Elle souligne notamment la nécessité d'établir des contrôles, en choisissant des échantillons de textes au hasard ou en élaborant des corpus parallèles. Finalement, Tymoczko se penche sur la question des modèles conceptuels en traductologie. Elle signale que ces derniers ont longtemps été basés sur des prémisses eurocentriques, prenant rarement en compte toute l'étendue du phénomène traduisant. Ainsi, tous les points qu'elle aborde au cours du chapitre lui servent à démontrer que la recherche empirique n'est pas objective en soi et qu'elle requiert de l'autoréflexion de la part du chercheur.

Après avoir vanté les mérites d'une reconceptualisation de la traduction, Tymoczko s'attaque dans la deuxième partie de son ouvrage à ce qu'elle juge être sa conséquence logique: la mise en valeur de l'agentivité (agency) des traducteurs et du pouvoir d'agir qui en résulte. Au chapitre 5, elle traite plus précisément d'un certain nombre de questions générales concernant l'agentivité politique des traducteurs. L'auteure présente d'abord ses propres travaux sur la Renaissance irlandaise. En prenant pour cadre la théorie postcoloniale, elle montre comment les traducteurs œuvrant dans un contexte d'asymétrie de pouvoir peuvent exercer leur agentivité en faisant des choix de traduction motivés par leur agenda politique, des choix que vont à l'encontre des normes dominantes; ces projets de traduction hautement politisés peuvent d'ailleurs engendrer des effets concrets au sein de la société où ils sont entrepris. Toutefois, elle insiste sur le fait que la théorie postcoloniale ne peut être utilisée comme cadre théorique pour étudier l'agentivité politique des traducteurs dans toutes les situations; on devrait limiter son application à des contextes vraiment postcoloniaux et élaborer de nouvelles approches théoriques pour les autres situations. En fait, Tymozcko affirme que, pour pouvoir étudier l'agentivité politique des traducteurs de façon générale, il faudrait formuler ce qu'elle appelle une théorie du pouvoir, capable 
de tenir compte de multiples facteurs: la lutte des classes, le commerce, le contrôle des ressources, la mondialisation, la culture corporative, les médias, les technologies, etc. Or, l'auteure affirme que les traducteurs n'ont heureusement pas besoin d'une telle théorie du pouvoir pour faire preuve d'agentivité politique dans leur pratique; elle croit néanmoins qu'une conceptualisation adéquate de l'activisme est, quant à elle, nécessaire. En effet, Tymoczko met de l'avant la métaphore de l'activisme comme engagement, métaphore qu'elle considère plus productive que celle avancée par Lawrence Venuti (activisme comme résistance). En supposant l'existence d'un ennemi spécifique, la métaphore de Venuti place selon elle les traducteurs dans une situation de réaction, en plus de donner lieu à des stratégies de résistance prescriptives. Au contraire, la métaphore de l'engagement implique une attitude proactive et permet aux traducteurs de choisir parmi plusieurs formes différentes d'activisme politique. Somme toute, l'auteure juge que les multiples choix auxquels font face les traducteurs dans leur pratique témoignent de leur agentivité; la pleine conscience de cette agentivité, à son tour, favorise leur pouvoir d'agir.

Tymoczko soutient toutefois que l'agentivité des traducteurs ne se limite pas à l'arène politique. En effet, à travers leurs choix de traduction, ces derniers peuvent aussi construire une image d'une culture tout entière: il s'agit de la traduction culturelle, dont elle discute au chapitre 6 . L'auteure débute en passant en revue les travaux de R. Jakobson, de J. C. Catford, de W. V. O. Quine et d'E. Nida, qu'elle juge néanmoins superficiels. En effet, elle explique que la notion de culture demeure mal définie en traductologie: on tend à ne s'intéresser qu'à des problèmes lexicaux découlant de divergences entre les réalités matérielles ou entre les pratiques de deux cultures. De plus, les traducteurs tendent à résoudre les problèmes d'incompatibilités culturelles ponctuellement, sans vision d'ensemble de la culture source. Pour mieux traduire la culture, Tymoczko propose donc de se référer à la théorie sociologique de P. Bourdieu, notamment à sa notion d'habitus. Elle va plus loin encore en déclarant qu'une approche holistique de la traduction culturelle est nécessaire. Une telle approche implique que le traducteur, avant d'entreprendre sa tâche, dresse un portrait de la culture source, qu'il en détermine l'habitus: sa vision de certains concepts clés (la liberté, la honte, le progrès, etc.), ses pratiques culturelles, ses paradigmes, ses surcodages (politesse, formes littéraires, etc.), etc. Selon Tymoczko, le traducteur se base ensuite sur ce portrait de la culture source pour élaborer une stratégie de traduction cohérente qui guidera ses choix et lui permettra d'atteindre ses buts (activisme politique ou idéologique, inno- vation littéraire, érudition, etc.). L'auteure est d'avis que cette approche holistique permet au traducteur de choisir en pleine connaissance de cause pourquoi et comment il traduit une culture donnée; il exerce ainsi son agentivité et s'autonomise par le fait même. En effet, selon Tymoczko, les traducteurs ne prennent pas assez de risques lorsqu'ils entreprennent une traduction culturelle, sousestimant la capacité de leurs destinataires à tolérer la nouveauté - l'Étranger - et à apprendre d'elle; ils minent ainsi leur propre agentivité. Somme toute, l'auteure souligne que les traducteurs font voir à leur public cible une culture qu'ils ont eux-mêmes construite à partir de leurs choix de traduction; ils sont donc d'importants médiateurs culturels.

Au chapitre 7, Tymoczko explore un autre domaine où les traducteurs peuvent faire preuve d'agentivité: le domaine du sens. Elle débute en remettant en question la vision traditionnelle du rôle du traducteur, selon laquelle ce dernier doit rendre le sens de l'original dans sa traduction. Cependant, l'auteure fait remarquer que le sens est une notion problématique en soi. Pour illustrer son argument, elle présente les résultats de deux exercices qu'elle propose souvent à ses étudiants en traduction; elle invite d'ailleurs le lecteur à y participer. Le premier exercice consiste à traduire librement un court poème médiéval irlandais. Tymoczko fait remarquer que toutes les versions produites par ses étudiants sont radicalement différentes les unes des autres, en terme d'interprétation du message, de choix lexicaux ou de la forme littéraire adoptée. Selon elle, cet exercice montre que le sens est construit par les traducteurs. Pour le second, il s'agit d'une session remue-méninges, durant laquelle les étudiants devaient dresser une liste de tous les éléments qui, à leur avis, constituent le sens d'un texte. Chaque groupe d'étudiants a réussi à en identifier un grand nombre: contenu, fonction, forme, grammaire, prosodie, éléments narratifs, réaction des lecteurs, méthode de production (médium, commercialisation, tirage...), etc. Le sens d'un texte est donc difficile à cerner. Ainsi, le traducteur doit nécessairement faire des choix et construire un sens nouveau en langue cible. Cette réalité permet d'ailleurs au traducteur d'exercer son agentivité. La conservation du sens ne peut donc pas être le but de la traduction. L'auteure est d'avis que les traducteurs doivent reconnaître cette réalité; ils pourraient alors faire leurs choix de traduction en pleine connaissance de cause et, ultimement, de façon éthique.

Tout au long de son livre, Tymoczko martèle le point de vue suivant: la traduction est un concept en grappe interculturel qui doit être revu et repensé pour aller au-delà des paradigmes occidentaux dominants qui l'ont régie par le passé. La définition conventionnelle de la traduction demeure 
trop exclusive; au contraire, elle devrait devenir ouverte et fluide pour permettre une interface entre de nombreuses cultures différentes. Une telle reconceptualisation engendrerait plusieurs changements au sein de la discipline: 1) la formulation de nouveaux cadres théoriques; 2) des méthodologies plus sophistiquées; 3) une pédagogie moins prescriptive; et surtout, 4) la prise de conscience des traducteurs et la mise en valeur de leur pouvoir d'agir. Cependant, si ce dernier point se concrétise, Tymoczko rappelle que, en exerçant leur agentivité, les traducteurs ne font pas nécessairement des choix éthiques. En effet, au chapitre 8 , elle souhaite que l'élargissement conceptuel qu'elle propose mènera à une étude plus approfondie des responsabilités des traducteurs et de l'éthique en traduction. Ceci doit être fait, insistet-elle, de manière à libérer les traducteurs plutôt que de leur prescrire des protocoles d'éthique rigides. Lauteure maintient que la formation des traducteurs devrait inclure un volet d'éthique contemporaine, où l'on encouragerait l'autoréflexion. Les traducteurs pourraient donc être en mesure d'identifier les facteurs pouvant influer sur leurs choix de traduction. De manière plus générale, l'auteure est d'avis que la discipline doit cesser de favoriser une éthique basée uniquement sur la fidélité microtextuelle, sur la responsabilité envers l'employeur et sur la soi-disant neutralité du traducteur; il faut plutôt reconnaitre l'importance des choix des traducteurs et encourager leur autoréflexion. Tymoczko croit que les traducteurs, ainsi sensibilisés à l'éthique, sauront passer outre les cadres culturels eurocentriques dominants et promouvoir la diversité et la différence.

Ce dernier ouvrage de Tymoczko n'est pas sans défaut. Lauteure relève de nombreux problèmes au sein de la traductologie et de la traduction professionnelle qui méritent effectivement d'être examinés. Toutefois, malgré la longueur du livre, elle soulève plusieurs questions mais offre peu de réponses définitives. Après avoir consulté l'ouvrage, le lecteur se trouve en effet soudainement conscient d'une myriade de facteurs dont il doit tenir compte en traduisant ou en menant à bien un projet de recherche, sans trop savoir comment y parvenir. Par exemple, la théorie du pouvoir dont l'auteure souhaite l'élaboration s'avèrerait être un appareil théorique particulièrement lourd. Il en va de même pour son approche holistique de la traduction culturelle, dont les exigences sont peu réalistes dans un contexte professionnel. Ainsi, en mettant l'emphase sur la rectitude théorique, elle sacrifie la faisabilité. De plus, dans sa volonté d'élargir le concept de traduction en se montrant la plus inclusive possible, l'auteure en vient de masquer la spécificité de certains types de traduction. Bien qu'elle affirme que ses propos lui sont applicables, elle dit bien peu de choses par rapport à la traduction spécialisée. En effet, sans vouloir remettre en cause la vision constructiviste du sens que Tymoczko met de l'avant, on ne peut nier que le rapport au sens dans ces domaines de spécialités diffère de celui que l'on observe en littérature, du fait de la présence d'un référent objectif qui restreint l'éventail des choix de traduction.

Cependant, Tymoczko demeure fidèle à sa réputation d'auteure au style élégant, limpide et efficace, dépourvu de jargon. En traçant son parcours personnel et professionnel dans l'introduction, elle met en relief avec franchise sa propre subjectivité, chose que peu de théoriciens avant elle ont eu le courage de faire. Elle invite également le lecteur à réfléchir et à s'adonner lui-même aux exercices qu'elle propose. Jamais dogmatique, elle livre ses opinions comme telles et prend soin de présenter les deux côtés de la médaille pour un problème donné. Tout au long de son livre, elle fait preuve d'ouverture en abordant un large éventail de sujets et de problématiques. De plus, l'auteure partage son temps également entre la théorie et la pratique de la traduction, leur accordant autant de valeur. Il est évident que, pour elle, la recherche en traduction doit profiter aux traducteurs euxmêmes; réciproquement, les traducteurs doivent reconnaître l'importance de la théorie pour leur pratique. Tymoczko a donc le mérite de rapprocher traducteurs et traductologues. Pour toutes ces raisons, son livre, dont la rigueur intellectuelle est indéniable, se distingue des autres publications universitaires au style souvent moins accessible. Ce faisant, Tymoczko reste cohérente avec le propos qu'elle défend, c'est-à-dire l'ouverture vers l'autre et la reconnaissance de la multiplicité des perspectives.

Eve-Marie Gendron-Pontbriand Université de Montréal, Montréal, Canada

\section{NOTES}

1. Nous avons choisi de traduire empowerment par «pouvoir d'agir». Voir la justification donnée dans: Le Bossé, Yann (2003): De «l'habilitation» au "pouvoir d'agir»: vers une appréhension plus circonscrite de la notion d'empowerment. Nouvelles pratiques sociales. 16(2):30-51.

2. L'astérisque est utilisé par l'auteure pour dénoter un concept en grappe. 